\title{
Reduced Multiplicative Tolerance Ranking and Applications
}

\author{
Sebastian Sitarz \\ Institute of Mathematics, University of Silesia, Katowice, Poland \\ ssitarz@ux2.math.us.edu.pl
}

\begin{abstract}
In this paper a reduced multiplicative tolerance - a measure of sensitivity analysis in multiobjective linear programming (MOLP) is presented. By using this new measure a method for ranking the set of efficient extreme solutions is proposed. The idea is to rank these solutions by values of the reduced tolerance. This approach can be applied to many MOLP problems, where sensitivity analysis is important for a decision maker. In the paper, applications of the presented methodology are shown in the market model and the transportation problem.
\end{abstract}

Index Terms - Multi-Criteria Analysis, Sensitivity Analysis, Multi-Objective Linear Programming, Decision Making

\section{Introduction}

The paper presents a method for ranking the efficient extreme solutions in MOLP problem by using sensitivity analysis. Here, the reduced multiplicative tolerance, a measure of sensitivity is proposed and used it in the ranking method. An inspiration to create this measure is an idea of combination of two approaches: the multiplicative tolerance approach and the standard approach. The reduced multiplicative tolerance is applied as a decision tool in multi-criteria problems. The chosen efficient solutions are ranked by the values of the reduced multiplicative tolerance. The greater value of the reduced tolerance there is, the higher position in ranking is obtained. The recommended solution is the first point in the obtained ranking.

The paper addresses the sensitivity analysis of efficient solutions in MOLP, thus other works on sensitivity in MOLP are worth mentioning as well. One of the first works is paper by Benson [2] who considers parameterization of the objective functions. A similar approach to Benson's is given by Sitarz [18]. Hansen et al. [8] consider the tolerance approach by means of weighted sum of objective functions. The general case of the tolerance approach (without weighting) is analysed by Hladik and Sitarz [11]. Comparison of the tolerance approach and the standard approach is given by Sitarz [15]. An interesting application of sensitivity analys is in Oly mpics rankings is given by Sitarz [17].
Chanas and Kuchta [3] present the MOLP problems with the interval objective coefficients.

The paper consists of the following sections: section I presents introduction; section II gives the basic notations and definitions; section III introduces a reduced multiplicative tolerance; section IV provides the computational aspects; section $\mathrm{V}$ presents a method of ranking the efficient extreme points in MOLP; section VI shows applications and the final section summarizes the paper.

\section{Notation and Basic Facts}

We consider the following MOLP problem:

$$
\operatorname{VMax}\{C x: x \in X\}
$$

where $C \in \mathbb{R}^{n, k}$ is a coefficients matrix of the objective functions $f_{i}(x)=c^{i} x \quad(i=1, \ldots, n), X \in \mathbb{R}^{n}$ is a set of feasible solutions defined by a matrix $A \in \mathbb{R}^{n, m}$ and a vector $b \in \mathbb{R}^{m}$ in the following way $X=\left\{x \in \mathbb{R}^{n}: A x \leq b, x \geq 0\right\}$. A feasible solution $x^{*} \in X$ is called an efficient solution to (1) if there is no $x \in X$ such that

$$
C x^{*} \leq C x \wedge C x^{*} \neq C x
$$

In the next sections, we focus on the efficiency of a given feasible solution $x^{*} \in X$ in a case of changing matrix $C$.

\subsection{Multiplicative tolerance approach}

The concept of sensitivity analysis by means of tolerance comes form Wendell [20]. Hladik in [9] considers tolerance in MOLP. The multiplicative tolerance approach aims to find a value (tolerance) representing the percentage perturbation which can be applied simultaneously to objective functions without affecting the efficiency. To define this formally, we introduce an additive $\delta$-neighbourhood of matrix $C=\left[c_{i j}\right]$ :

$$
O_{\delta}(C)=\left\{D=\left[d_{i j}\right]:\left|d_{i j}-c_{i j}\right| \leq \delta\left|c_{i j}\right|\right\}
$$

We consider a new problem - reformulated problem (1) by matrix $D \in O_{\delta}(C)$ :

$$
\operatorname{VMax}\{D x: x \in X\}
$$


Definition 1. An multiplicative tolerance for an efficient solution $x^{*}$ is any real $\delta$ such that $x^{*}$ remains efficient to (2) for all $D \in O_{\delta}(C)$. The maximal multiplicative tolerance is denoted by $\delta_{\max }$.

Remark 1. Some interesting properties of the tolerance are given in works $[9,10,11]$. We will focus on the tolerance and its modification in the next sections of this paper.

\subsection{Standard approach}

The approach to sensitivity analysis, presented in this subsection, comes from the early linear programming theory. The use of this approach in MOLP was proposed by Sitarz [14]. The standard approach, called the standard sensitivity analysis aims to find the values (a parameter set) of a selected one objective function coefficient which can be applied without affecting the efficiency. To define it formally, let us introduce a new matrix $D_{t}=\left[d_{i j}\right]$ which is obtained from matrix $C$ by changing only one coefficient into parameter $t$ :

$$
d_{i j}= \begin{cases}c_{i j}, & \text { if }(i, j) \neq(q, p) \\ t, & \text { if }(i, j)=(q, p)\end{cases}
$$

We consider a new problem- reformulated problem (1) by matrix $D_{t}$ :

$$
\operatorname{VMax}\left\{D_{t} x: x \in X\right\}
$$

Definition 2. The set of parameters $t$, for which $x^{*}$ is an efficient solution to (3) is denoted by $T_{x^{*}}$

Remark 2. Set $T_{x^{*}}$ is an interval (it might not be a closed interval, [14]), for wider consideration of closeness look at [16]). Thus, let us introduce the following notation:

$$
c l T_{x^{*}}=\left[c_{q p}-\underline{\delta}_{q p}, c_{q p}+\bar{\delta}_{q p}\right]
$$

where $\delta_{q p}, \bar{\delta}_{q p} \in[0, \infty]$ are the maximal lower and upper perturbations of $c_{q p}$. Moreover, let us notice simple consequence of the above facts:

$$
\left(c_{q p}-\underline{\delta}_{q p}, c_{q p}+\bar{\delta}_{q p}\right) \subset T_{x^{*}} \subset\left[c_{q p}-\underline{\delta}_{q p}, c_{q p}+\bar{\delta}_{q p}\right]
$$

\section{Reduced Multiplicative Approach}

The aim of this section is to present a measure which combines the tolerance approach (subsection II.A) and the standard approach (subsection II.B). The idea of the proposed approach is to put the coefficients into two groups:

The first group consists of the coefficients which can be changed - these coefficients change their values over the initial values.

The second group consists of the coefficients which can not be changed - these coefficients are equal to the initial values.
To present the above idea formally, we introduce the following notations and definitions. Let $I$ be an index set of the changed coefficients, then we have $I \subset$ $\{1, \ldots, k\} \times\{1, \ldots, n\}$. Let us introduce an additive $\delta$ neighbourhood of matrix $C=\left[c_{i j}\right]$ including only the coefficients from set $I$ :

$$
\begin{gathered}
O_{\delta}^{I}(C)=\left\{D^{I}=\left[d_{i j}\right]:\left|d_{i j}-c_{i j}\right| \leq \delta\left|c_{i j}\right| \wedge \quad d_{i j}\right. \\
\left.=c_{i j} \text { for }(i, j) \notin I\right\}
\end{gathered}
$$

We consider a new problem - reformulated problem (1) by matrix $D^{I} \in O_{\delta}^{I}(C)$ :

$$
\operatorname{VMax}\left\{D^{I} x: x \in X\right\}
$$

Definition 3. A reduced multip licative $I$-tolerance for an efficient solution $x^{*}$ is any real $\delta$ such that $x^{*}$ remains efficient to (4) for all $D^{I} \in O_{\delta}^{I}(C)$. The maximal reduced multiplicative $I$-tolerance is denoted by $\delta_{\max }^{I}$.

The following theorem presents the properties of the maximal reduced multiplicative $I$-tolerance.

Theorem 1.

a) The connection between $\delta_{\max }^{I}$ and the multiplicative tolerance approach has the following form:

$$
I=\{1, \ldots, k\} \times\{1, \ldots, n\} \Rightarrow \delta_{\text {max }}^{I}=\delta_{\text {max }}
$$

b) The greater set $I$ there is the smaller value of $\delta_{\text {max }}^{I}$ is obtained:

$$
I \subset J \Rightarrow \delta_{\max }^{J} \leq \delta_{\max }^{I}
$$

c) The connection between $\delta_{\max }^{I}$ and the standard approach has the following form:

$$
I=\{(q, p)\} \Rightarrow \delta_{\text {max }}^{I}=\frac{1}{\left|c_{q p}\right|} \min \left\{\underline{\delta}_{q p}, \bar{\delta}_{q p}\right\}
$$

d) The lower and upper bounds of $\delta_{\max }^{I}$ have the following form:

$$
(q, p) \in I \Rightarrow \delta_{\text {max }} \leq \delta_{\text {max }}^{I} \leq \frac{1}{\left|c_{q p}\right|} \min \left\{\underline{\delta}_{q p}, \bar{\delta}_{q p}\right\}
$$

Proof

a) Let $I=\{1, \ldots, k\} \times\{1, \ldots, n\}$, then $I^{\prime}=\varnothing$ and

$$
O_{\delta}^{I}(C)=\left\{D^{I}=\left[d_{i j}\right]:\left|d_{i j}-c_{i j}\right| \leq \delta\left|c_{i j}\right|\right\}
$$

Hence

$$
O_{\delta}^{I}(C)=O_{\delta}(C)
$$

Thus, $\delta$ is the reduced multiplicative $I$-tolerance for $x^{*}$ if and only if $\delta$ is the multiplicative tolerance for $x^{*}$.

Hence, by using definitions 1 and 3 , we have

$$
\delta_{\max }^{I}=\delta_{\text {max }}
$$

b) If $I \subset J$ then $O_{\delta}^{J}(C) \subset O_{\delta}^{I}(C)$. Indeed, let $D \subset O_{\delta}^{J}(C)$. Then 


$$
d_{i j}=c_{i j} \text { for }(i, j) \notin J
$$

Since $J^{\prime} \subset I^{\prime}$ we obtain that

$$
d_{i j}=c_{i j} \text { for }(i, j) \notin I
$$

Moreover, since $I \subset J$ we have for $(i, j) \in I \subset J$

$$
\left|d_{i j}-c_{i j}\right| \leq \delta\left|c_{i j}\right|
$$

We have shown that $O_{\delta}^{J}(C) \subset O_{\delta}^{I}(C)$.

Using the above fact, we obtain that if $x^{*}$ remains efficient to (4) for all $D \subset O_{\delta}^{J}(C)$ then $x^{*}$ remains efficient to (4) for all $D \subset O_{\delta}^{I}(C)$. Thus, if $\delta$ is a reduced $J$-tolerance for $x^{*}$, then $\delta$ is a reduced $I$ tolerance for $x^{*}$. Hence, by using definition 3 , we have

$$
\delta_{\max }^{J} \leq \delta_{\max }^{I}
$$

c) In the case of $I=\{(q, p)\}$ we have

$$
\begin{gathered}
O_{\delta}^{I}(C)=\left\{D^{I}=\left[d_{i j}\right]:\left|d_{q p}-c_{q p}\right|<\delta\left|c_{q p}\right| \wedge d_{i j}\right. \\
\left.=c_{i j} \text { for }(i, j) \neq(q, p)\right\}
\end{gathered}
$$

Thus, $x^{*}$ remains efficient to (4) for all $D^{I} \in O_{\delta}^{I}(C)$ if and only if $x^{*}$ is efficient to (2) for $t=d_{q p}$ and

$$
\left|t-c_{q p}\right|<\delta\left|c_{q p}\right|
$$

Thus

$$
\begin{aligned}
O_{\delta}^{I}(C)=\left\{D^{I}=[\right. & \left.d_{i j}\right]:\left|t-c_{q p}\right| \\
& <\delta\left|c_{q p}\right| \wedge \quad t \in T_{x^{*}} \wedge d_{i j} \\
& \left.=c_{i j} \text { for }(i, j) \neq(q, p)\right\}
\end{aligned}
$$

where $d_{q p}=t$.

Moreover, by using remark 2 we obtain the fact that $t \in T_{x^{*}}$ and $\left|t-c_{q p}\right|<\delta\left|c_{q p}\right|$ if and only if

$$
\delta<\frac{1}{\left|c_{q p}\right|} \underline{\delta}_{q p} \text { and } \delta<\frac{1}{\left|c_{q p}\right|} \bar{\delta}_{q p}
$$

Thus, by using definition 3 we obtain the fact that:

$$
\delta_{\max }^{I}=\frac{1}{\left|c_{q p}\right|} \min \left\{\underline{\delta}_{q p}, \bar{\delta}_{q p}\right\}
$$

d) If $(q, p) \in I$, then $\{(q, p)\} \subset I$, thus by using point $b$ ) we obtain the fact that:

$$
\delta_{\max }^{I} \leq \delta_{\max }^{\{(q, p)\}}
$$

By using point c) we have:

$$
\delta_{\max }^{I} \leq \frac{1}{\left|c_{q p}\right|} \min \left\{\underline{\delta}_{q p}, \bar{\delta}_{q p}\right\}
$$

Moreover $I \subset\{1, \ldots, k\} \times\{1, \ldots, n\}$, thus by using point $b$ ) we obtain the fact that:

$$
\delta_{\max }^{\{1, \ldots, k\} \times\{1, \ldots, n\}} \leq \delta_{\max }^{I}
$$

By using point a) we have:

$$
\delta_{\max } \leq \delta_{\max }^{I}
$$

In this way, we have shown that:

$$
\delta_{\text {max }} \leq \delta_{\text {max }}^{I} \leq \frac{1}{\left|c_{q p}\right|} \min \left\{\underline{\delta}_{q p}, \bar{\delta}_{q p}\right\}
$$

Remark 3. The following points are comments to the corresponding points of theorem 1 .

a) The connection between the reduced multip licative $I$-tolerance and the multiplicative tolerance looks as follows: In the special case of the reduced multip licative $I$-tolerance (for $I=\{1, \ldots, k\} \times\{1, \ldots, n\}$,) we obtain the maximal multiplicative tolerance. This case corresponds to the case of changing all coefficients.

b) If we change more coefficients then the maxima reduced multiplicative $1 I$-tolerance is lower. In other words, the more changes there are the less sensitivity is obtained.

c) The connection between the reduced multiplicative I-tolerance and the standard approach looks as follows: In the special case of the reduced multiplicative $I$ tolerance (for $I$ consisting of only one given element) we obtain case very similar to the standard approach. This case corresponds to the case of changing only one coefficient.

d) The maximal reduced multiplicative $I$-tolerance is a value between the optimal values obtained by the multiplicative tolerance and the standard approach.

We have defined and we have given properties of the reduced multiplicative $I$-tolerance. Now we are going to describe the methods of computation the maximal reduced multiplicative $I$-tolerance (section IV).

\section{Methods of Computation}

After presenting the theoretical background of the reduced tolerance we focus on the methods of computation the maximal reduced tolerance. In the proposed methods we use the known algorithms presented in the earlier papers. We divide the presented methods into groups depending on the number of the changed coefficients. As we can see below, in the special cases it is easy to calculate the maximal reduced tolerance, but in general the problem of finding the maximal reduced tolerance is NP-hard.

\subsection{The case of changing one coefficient}

The case of changing one coefficient $(I=(q, p))$ was considered in [14]. We can use point c) of theorem 1 :

$$
\delta_{\max }^{I}=\frac{1}{\left|c_{q p}\right|} \min \left\{\underline{\delta}_{q p}, \bar{\delta}_{q p}\right\},
$$

and already presented characterization of interval $T_{x^{*}}$ :

$$
\operatorname{cl} T_{x^{*}}=\left[c_{q p}-\underline{\delta}_{q p}, c_{q p}+\bar{\delta}_{q p}\right]
$$

Thus, to find the maximal reduced multiplicative $I$ tolerance $\delta_{\max }^{I}$, set $T_{x^{*}}$ can be used. The detailed description of the algorithm for finding $\operatorname{set} T_{x^{*}}$. is given 
in work by Sitarz [14]. This algorithm is based on the analysis of the simplex tableau $\mathrm{x}$ and uses works by Steuer [19] and Gal [6]. This approach is easy to apply and needs solving some linear inequalities. On the other hand, we can use more general method presented by Sitarz in [18]. This method uses parametric linear programming problems for obtaining $\underline{\delta}_{q p}$, and $\bar{\delta}_{q p}$. This algorith $m$ is easy to apply and needs only a software to one-criterion parametric linear programming problems.

\subsection{The general case}

The general case of set $I$ corresponds to the multiplicative tolerance. In details this case was considered in the paper by Hladik and Sitarz [11] where it was shown that the problem of determining the maximal tolerance is NP-hard. In spite of NP-hardness, Hladik and Sitarz [11] propose the sequence of problems to obtain the maximal tolerance.

\section{The REMTOR Method}

For the MOLP problem (1) the decision maker has to choose an efficient solution from the set of efficient extreme points. This problem was considered by many researches, for example by Steuer [19] and Zeleny [22]. Here, a method of ranking the efficient extre me points of MOLP problem is going to be presented. The first ranked point is the recommended solution of this problem. The idea of the presented method is to use the values of maximal reduced tolerance for the efficient extreme points. As we know, the bigger value of the maximal reduced tolerance there is, the less sensitivity is obtained. This approach can be applied as a decision tool to a multi-criteria problem as well. The algorithm of the method consists of the following steps.

Step 1. Formulation of the multi-criteria problem in a form of MOLP problem (description of problem (1), see section 2).

Step 2. Describing the coefficients which can be changed (description of set $I$, see section 3 ).

Step 3. Finding all the efficient extreme solutions (by the ADBASE method, [19] or similar algorithms: [5], [21].

Step 4. Calculating the maximal reduced multiplicative $I$-tolerance for the efficient extreme solutions (see sections 4 and 5).

Step 5. Constructing the ranking of the efficient extreme solutions by values of the maximal reduced $I$ tolerance (the bigger value of the maximal reduced tolerance there is the higher position in the ranking is obtained).

The above procedure will be given a name of the REMTOR method (from Reduced Multiplicative Tolerance Ranking).

\subsection{Illustrative example of the REMTOR method}

To illustrate the REMTOR method, we consider a problem presented earlier by Gass and Roy [7] problem (6).

Step 1. The MOLP problem has the following form:

$$
\begin{gathered}
\operatorname{VMax}\left[\begin{array}{l}
x_{1} \\
\\
x_{2}
\end{array}\right] \\
x_{1}+x_{2} \leq 8 \\
2 x_{1}+x_{2} \leq 12 \\
x_{1}+2 x_{2} \leq 14 \\
9 x_{1}+7 x_{2} \leq 63 \\
-4 x_{1}+10 x_{2} \leq 61 \\
2 x_{1}-x_{2} \leq 8 \\
14 x_{1}+3 x_{2} \leq 72 \\
x_{1}, x_{2} \geq 0
\end{gathered}
$$

Step 2. Let us assume that all the coefficients can be changed. It means that

$$
I=\{(1,1),(1,2),(2,1),(2,2)\} .
$$

Step 3. We obtain the following six efficient extreme solutions:

$$
\begin{gathered}
x^{1}=[4.8,1.6], x^{2}=[4.5,3], x^{3}=[4.2,3.6], \\
x^{4}=[3.5,4.5], x^{5}=[2,6], x^{6}=[1,6.5] .
\end{gathered}
$$

Step 4. We calculate the maximal reduced $I$ tolerance for all the efficient extreme solutions and obtain the tolerances described in table 1 .

Step 5. We rank the efficient extreme solutions with respect to the values of maximal reduced tolerance and obtain the results in table 1 . Moreover, in this table we present values of the objective function and values of the maximal reduced $I$-tolerance. As we can see in table 1 , the recommended solutions are $x^{4}$ and $x^{5}$ as the efficient extreme solutions with the biggest tolerance.

Table 1: The ranking obtained by the REMTOR method for $I=\{(1,1),(1,2),(2,1),(2,2)\}$

\begin{tabular}{|c|c|c|}
\hline Ranking & Efficient Solutions & $\boldsymbol{\delta}_{\max }^{I}$ \\
\hline 1 & $x^{5}$ & 0.667 \\
\hline 2 & $x^{4}$ & 0.500 \\
\hline 2 & $x^{3}$ & 0.500 \\
\hline 4 & $x^{2}$ & 0.333 \\
\hline 5 & $x^{6}$ & 0.300 \\
\hline 6 & $x^{1}$ & 0.222 \\
\hline
\end{tabular}

\subsection{Influence of set I on the REMTOR method}

Let us notice that ranking obtained by the REMTOR method depends on set $I$. To illustrate this observation, 
we consider the same problem as in subsection 5.1 with only one change - set $I$ has the following form:

$$
I=\{(1,1),(1,2),(2,2)\} \text {. }
$$

Table 2 presents ranking obtained in this case. As we can see in table 2 , the recommended solution is $x^{6}$ as the efficient extreme solution with the biggest tolerance.

Table 2: The ranking obtained by the REMTOR method for $I=\{(1,1),(1,2),(2,2)\}$.

\begin{tabular}{|c|c|c|}
\hline Ranking & Efficient Solutions & $\boldsymbol{\delta}_{\max }^{I}$ \\
\hline 1 & $x^{6}$ & 0.916 \\
\hline 2 & $x^{5}$ & 0.888 \\
\hline 3 & $x^{4}$ & 0.667 \\
\hline 3 & $x^{3}$ & 0.667 \\
\hline 5 & $x^{2}$ & 0.500 \\
\hline 6 & $x^{1}$ & 0.417 \\
\hline
\end{tabular}

By comparing tables 1 and 2, we can see that the rankings differ. The reason is obvious - the set of changed coefficients is different. In practice, this set is given by the decision maker. Thus, the final form of ranking depends on the choice of the decision maker.

\section{Applications}

\subsection{Application in the market model}

The model presented below is taken from the work of Ekeland [4]. The presented market model refers to the general economic theory. In our model there is a group of agents. One can purchase a certain amount of goods on the market. The agent's aim is to reach the greatest satisfaction from the purchase of goods. The satisfaction is measured by the utility function. The aim of the problem is to find an allocation of goods among the agents. The description of the above model is given below.

$n$ - the number of goods on the market.

$m-$ the number of agents.

$\Omega_{\mathrm{i}}$ - value of $i$-th good available on the market, $\Omega_{\mathrm{i}} \in$ $\mathbb{R}$, for $i \in\{1, \ldots, n\}$.

$u_{k}: \mathbb{R}^{n} \rightarrow \mathbb{R}$ - utility function of $k$-th agent.

In the presented model, we assume that all utility functions are linear. The feasible allocations are the vectors

$$
x=\left(x_{1}^{1}, \ldots, x_{n}^{1}, \ldots \ldots, x_{1}^{m}, \ldots, x_{n}^{m}\right) \in \mathbb{R}_{+}^{n \cdot m}
$$

which for all $i \in\{1, \ldots, n\}$ satisfy the following condition:

$$
\sum_{k=1}^{m} x_{i}^{k}=\Omega_{\mathrm{i}}
$$

The real number $x_{i}^{k}$ denotes the value of $i$-th good which is assigned to the $k$-th agent. Thus, the feasible allocations describe the ways of distribution of goods among the agents. The set of all feasible allocations is denoted by $A$.

The feasible allocation $x=\left(x_{1}^{1}, \ldots, x_{n}^{1}, \ldots \ldots, x_{1}^{m}, \ldots, x_{n}^{m}\right) \in A$ is connected with the values of the utility function of all agents:

$$
u(x)=\left(u_{1}\left(x_{1}^{1}, \ldots, x_{n}^{1}\right), \ldots \ldots, u_{m}\left(x_{1}^{m}, \ldots, x_{n}^{m}\right)\right)
$$

The feasible allocation $x \in A$ is said to be the Pareto optimal allocation if there is no allocation $y \in A$ such that

$$
u(x) \leq u(y) \text { and } u(x) \neq u(y)
$$

The initial problem of the market model is to find all Pareto optimal allocations or its subset. Furthermore the analysis of the Pareto optimal allocations is needed to choose the best allocation. It can be done by means of the sensitivity analysis of the initial data and it will be presented in next parts of this section.

The problem of finding the Pareto optimal allocations can be written down in the form of MOLP problem in the following way:

$$
\begin{gathered}
\operatorname{VMax}\left[u_{1}\left(x_{1}^{1}, \ldots, x_{n}^{1}\right), \ldots \ldots, u_{m}\left(x_{1}^{m}, \ldots, x_{n}^{m}\right)\right] \\
\sum_{k=1}^{m} x_{i}^{k}=\Omega_{\mathrm{i}}, \text { for all } i \in\{1, \ldots, n\} \\
x_{1}^{1}, \ldots, x_{n}^{1}, \ldots \ldots, x_{1}^{m}, \ldots, x_{n}^{m} \geq 0
\end{gathered}
$$

The efficient allocations of problem (7) are the Pareto optimal allocations. Moreover, in the MOLP problem (7) we have $m$ criteria connected with the agents and $m \cdot n$ decision variables connected with the coefficients of the allocation. We proceed the REMTOR method for the market model.

Step 1. Let us consider the following market model:

- $m=2$ - there are 2 agents on the market,

- $n=4$ - there are 4 goods to distribute,

- $\Omega_{1}=1, \Omega_{2}=1, \Omega_{3}=1, \Omega_{4}=2-$ the values of goods available on the market.

The agents have the following utility functions:

- $u_{1}\left(x_{1}^{1}, x_{2}^{1}, x_{3}^{1}, x_{4}^{1}\right)=4 x_{1}^{1}+3 x_{2}^{1}+2 x_{3}^{1}+x_{4}^{1}$

- $u_{2}\left(x_{1}^{2}, x_{2}^{2}, x_{3}^{2}, x_{4}^{2}\right)=x_{2}^{2}+2 x_{3}^{2}+2 x_{4}^{2}$

The MOLP problem connected with the above market model has the following form: 


$$
\begin{gathered}
\operatorname{VMax}\left[4 x_{1}^{1}+3 x_{2}^{1}+2 x_{3}^{1}+x_{4}^{1}, \quad x_{2}^{2}+\right. \\
\left.2 x_{3}^{2}+2 x_{4}^{2}\right] \\
x_{1}^{1}+x_{1}^{2}=1 \\
x_{2}^{1}+x_{2}^{2}=1 \\
x_{3}^{1}+x_{3}^{2}=1 \\
x_{4}^{1}+x_{4}^{2}=2 \\
x_{1}^{1}, x_{2}^{1}, x_{3}^{1}, x_{4}^{1}, x_{1}^{2}, x_{2}^{2}, x_{3}^{2}, x_{4}^{2} \geq 0
\end{gathered}
$$

Step 2. With no additional information, we allow to change all the coefficients of criteria function (the coefficients of utility functions).
Step 3. There are 5 efficient extreme solutions of the considered problem (8), see table 3.

Step 4. We calculate the maximal reduced tolerance for all the efficient extreme solutions and obtain the tolerances described in table 3 .

Step 5. We rank the efficient extreme solutions with respect to the values of maximal reduced tolerance (table 3). Using this ranking, we obtain the fact that the recommended solution is the first solution in this ranking. Furthermore, by using the maximal reduced tolerance for this solution, we can say that all coefficients of the utility functions may vary simultaneously $15,4 \%$ without losing efficiency.

Table 3: The ranking obtained by the REMTOR method in the market model

\begin{tabular}{|c|c|c|c|}
\hline Ranking & Pareto Allocations & {$\left[\mathbf{u}_{\mathbf{1}}, \mathbf{u}_{2}\right]$} & $\boldsymbol{\delta}_{\max }^{\boldsymbol{I}}$ \\
\hline 1 & $(1,1,1,0,0,0,0,2)$ & $(9,4)$ & 0.154 \\
\hline 2 & $(1,1,0,0,0,0,1,2)$ & $(7,6)$ & 0.122 \\
\hline 3 & $(1,1,1,2,0,0,0,0)$ & $(11,0)$ & 0.101 \\
\hline 4 & $(1,0,0,0,0,1,1,2)$ & $(4,7)$ & 0.095 \\
\hline 5 & $(1,1,1,1,0,0,0,1)$ & $(10,2)$ & 0.087 \\
\hline
\end{tabular}

\subsection{Application in the transportation problem}

We consider the multi-criteria transportation problem. This problem is considered in many papers, for example: [13], [12], [1]. We proceed the REMTOR method for two criteria transportation problem.

Step 1. Let us consider the transportation problem from work by Pandian and Anuradha [13]. We have a transportation model of a company with three factories (F1, F2 F3), and four warehouses (W1, W2, W3, W 4).
A given product is transported from factories to warehouses. There are two criteria: the minimization of total transportation and the minimization of total product deterioration during transportation. The cost of the transportation and the deterioration cost of the

\begin{tabular}{|c|c|c|c|c|c|c|}
\hline \multirow{2}{*}{\multicolumn{2}{|c|}{ (cost, deteriorations) }} & \multicolumn{4}{|c|}{ Warehouses } & \multirow{3}{*}{$\begin{array}{c}\text { Supply } \\
8\end{array}$} \\
\hline & & \multirow{2}{*}{$\frac{\mathbf{W}_{\mathbf{1}}}{(1,4)}$} & \multirow{2}{*}{$\frac{\mathbf{W}_{\mathbf{2}}}{(2,4)}$} & \multirow{2}{*}{$\frac{\mathbf{W}_{\mathbf{3}}}{(7,3)}$} & \multirow{2}{*}{$\frac{\mathbf{W}_{4}}{(7,4)}$} & \\
\hline \multirow{3}{*}{ Factories } & $\mathrm{F}_{1}$ & & & & & \\
\hline & $\mathrm{F}_{2}$ & $(1,5)$ & $(9,8)$ & $(3,9)$ & $(4,10)$ & 19 \\
\hline & $\mathrm{F}_{3}$ & $(8,6)$ & $(9,2)$ & $(4,5)$ & $(6,1)$ & 17 \\
\hline \multicolumn{2}{|c|}{ Demand } & 11 & 3 & 14 & 16 & \\
\hline
\end{tabular}
product during transportation are given in table 4 . It is well known that such transportation problem can be formulated by means of linear programming, in this case by MOLP. Thus, we are able to use the REMTOR method in this problem.

Table 4: Data for the transportation problem 
Table 5: The ranking obtained by the REMTOR method in the transportation problem

\begin{tabular}{|c|c|c|c|c|c|}
\hline ranking & $\begin{array}{c}\text { Values of the } \\
\text { efficient solutions }\end{array}$ & $\boldsymbol{\delta}_{\text {max }}^{I}$ & ranking & $\begin{array}{c}\text { Values of the } \\
\text { efficient solutions }\end{array}$ \\
\hline 1 & $(176,175)$ & 0.205 & 12 & $(143,265)$ \\
\hline 2 & $(168,185)$ & 0.199 & 13 & $(153,215)$ \\
\hline 3 & $(197,169)$ & 0.197 & 14 & $(149,235)$ \\
\hline 4 & $(208,167)$ & 0.192 & 15 & $(150,230)$ \\
\hline 5 & $(172,180)$ & 0.182 & 16 & $(148,240)$ \\
\hline 6 & $(164,190)$ & 0.183 & 17 & $(146,250)$ \\
\hline 7 & $(186,171)$ & 0.181 & 18 & $(147,245)$ \\
\hline 8 & $(160,195)$ & 0.179 & 19 & 0.163 \\
\hline 9 & $(156,200)$ & 0.175 & 20 & $(144,260)$ \\
\hline 10 & $(154,210)$ & 0.174 & 21 & 0.157 \\
\hline 11 & $(155,205)$ & 0.172 & 22 & $(145,255)$ \\
\hline
\end{tabular}

Step 2. With no additional information, we allow all the coefficients to be changed.

Step 3. There are 22 efficient extreme solutions of this problem (for details see [13]). To clarify notation, we present these solutions in the criteria space (table 5).

Step 4 . We calculate the maximal reduced tolerance for all efficient extreme points and obtain tolerances described in table 5 .

Step 5. We rank the efficient points with respect to the values of maximal reduced tolerance (table 5). Using this ranking, we obtain the fact that the recommended solution is the first solution in this ranking. Furthermore, by using the maximal reduced tolerance for this solution, we can say that all costs and deteriorations may vary simultaneously $20,5 \%$ while retaining efficiency of this solution.

\section{Conclusion}

In this paper a method for ranking the efficient extreme points in MOLP problems - the REMTOR method is presented. The presented REMTOR method has a form of ranking the efficient extreme points in MOLP. These points are ranked by values of the reduced tolerance. The bigger value of the reduced multiplicative tolerance there is, the higher position in ranking is obtained. This approach can be applied in many MOLP problems where the sensitive analys is is important for the decision maker. It is worth noticing that it is possible to use the REMTOR method in a wider class of multi-criteria problems. If we are able to compute the maximal reduced tolerance in a given multi-criteria problem, then we are able to use the REMTOR method.

The REMTOR method was based on the reduced multiplicative tolerance - a measure of sensitivity in MOLP with respect to perturbation of the objective functions coefficients. The idea of the proposed measure was putting the coefficients into two groups: the first group consisted of the coefficients, which could be changed and the second group consisted of the coefficients which could not be changed. In other words, we reduced the number of changed coefficients in the multiplicative tolerance approach, thus we this new measure was given the name of the reduced tolerance. Some properties of the maximal reduced multiplicative tolerance were shown.

\section{References}

[1] Aneya Y. P., Nair P. K. (1979). Bicriteria transportation problem, Management Science 25 (1), 73-78.

[2] Benson H. P. (1985). Multiple objective linear programming with parametric criteria coefficients. Management Science 31 (4), 461-474.

[3] Chanas, S., Kuchta, D. (1996), Multiobjective programming in optimization of interval objective functions a generalized approach, European Journal of Operational Research 94, 594-598.

[4] Ekeland I. (1979). Elements d'economic mathematic, Hermann, Paris.

[5] Evans, J.P., Steuer, R.E. (1973). A revised simplex method for linear multiple objective programs. Mathematical Programming 5, 54-72.

[6] Gal T. (1995). Postoptimal Analyses, Parametric Programming and Related Topics. Walter de Gruyter, Berlin.

[7] Gass S. I., Roy P. G. (2003). The compromise hypersphere for multiobjective linear programming, European Journal of Operational Research 144 , 459-479. 
[8] Hansen P., Labbe M., Wendell R.E. (1989). Sensitivity Analysis in Multiple Objective Linear Programming: The Tolerance Approach. European Journal of Operational Research 38, 63-69.

[9] Hladik M. (2008). Computing the tolerances in multiobjective linear programming. Optimization. Methods \& Software, 23 (5), 731-739.

[10] Hladik M. (2008). Additive and multiplicative tolerance in multiobjective linear programming, Operations Research Letters 36, 393-396.

[11] Hladik M., Sitarz S. (2010). Maximal and supremal tolerances in multiobjective linear programming. Technical report KAM-DIMATIA, Series: 2010-989, Department of Applied Mathematics, Charles University, Prague.

[12] Murad, A., Al-Ali, A., Ellaimony, E., Abdelwali, H., (2010). On bi-criteria two-stage transportation problem: A case study. Transport Problems 5 (3), 103-114.

[13] Pandian P., Anuradha D. (2011). A new method for solving bi-objective transportation problems, Australian Journal of Basic and Applied Sciences, 5(10): 67-74.

[14] Sitarz S. (2008). Postoptimal analysis in multicriteria linear programming. European Journal of Operational Research. 191, 7-18.

[15] Sitarz S. (2010). Standard sensitivity analysis and additive tolerance approach in MOLP. Annals of Operations Research, 181(1), 219-232.

[16] Sitarz S. (2011). Sensitivity analysis of weak efficiency in multiple objective linear programming. Asia-Pacific Journal of Operational Research, 28(4), 445-455.

[17] Sitarz S. (2012). Mean value and volume-based sensitivity analysis for Olympic rankings, European Journal of Operational Research, 216, 232-238.

[18] Sitarz S. (2012). Parametric LP for sensitivity analysis of efficiency in MOLP problems, Optimization Letters, [in press], doi: 10.1007/s 11590-012-0541-1.

[19] Steuer R. (1986). Multiple Criteria Optimization Theory: Computation and Application. John Willey, New York.

[20] Wendell R. E. (1982) A preview of a tolerance approach to sensitivity analysis in linear programming, Discrete Mathemtaics, 38, 121-124.

[21] Yu, P.-L., Zeleny, M. (1975). The set of all nondominated solutions in linear cases and a multicriteria simplex method. Journal of Mathematical Analysis and Applications 49 (2), 430-468.
[22] Zeleny, M. (1982). Multiple Criteria Decision Making. McGraw-Hill Book Company, New York.

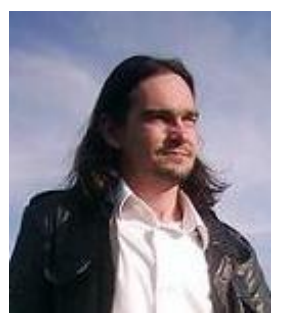

Sebastian Sitarz was born on March 19, 1975. He received his MS in Mathematics from University of Silesia (Poland) in 1999; $\mathrm{PhD}$ in Economics from University of Lodz in 2003 (Poland). Currently, he is working as a Lecturer in the Institute of Mathematics, the University of Silesia, Katowice (Poland), since 2003. His research areas include multiobjective linear programming, dynamic programming and their applications. His papers appeared in European Journal of Operational Research, Fuzzy Sets and Systems, Computers and Operations Research, Annals of Operations Research, Applied Mathematics and Computation and others.

How to cite this paper: Sebastian Sitarz,"Reduced Multiplicative Tolerance Ranking and Applications", International Journal of Intelligent Systems and Applications(IJISA), vol.5, no.3, pp.50-57, 2013.DOI: 10.5815/ijisa.2013.03.05 